\title{
A REVIEW ON VIBRATION MITIGATION OF BORING BAR USING PASSIVE DAMPING TECHNIQUES
}

\author{
Pooja J. Waghmare ${ }^{1}$, R. V. Patil ${ }^{2}$, G.S. Waghmare ${ }^{3}$ \\ ${ }^{I}$ P.G.Student, Department of Mechanical Engineering, Sinhgad Institute of Technology \& Science, Maharashtra, \\ India \\ ${ }^{2}$ Assistant Professor, Department of Mechanical Engineering, Sinhgad Institute of Technology \& Science, \\ Maharashtra, India \\ ${ }^{3}$ Assistant Professor, Department of Mechanical Engineering, Sinhgad Institute of Technology \& Science, \\ Maharashtra, India
}

\begin{abstract}
Work piece and tool vibration control has been a subject of primary importance in manufacturing industry. High slenderness ratio is a reason for boring bar to be subjected to high amount of vibrations that accelerate the tool wear affecting the tool life, higher noise level and increased deviations. This review focuses on various damping techniques such as passive damping systems using particle impact damping and Magneto-Rheological (MR) fluid for vibration attenuation purpose. This also introduces the mechanics of boring operation that affects the tool wear, surface roughness, noise and hence affects the productivity. This review compares the results of boring bar using passive damping and the conventional tool on the basis of modal analysis of vibrating structure, and the test results. This paper explains the vibration behavior of a conventional boring bar and a boring bar using passive techniques for vibration reduction.
\end{abstract}

Keywords: Damping particles, vibrations, Magneto-Rheological fluid (MR).

\section{INTRODUCTION}

The vibration between a tool and a workpiece is an important issue in machining operations, as it may deteriorate the workpiece surface quality, lower the productivity, and shorten the tool life. Boring process is an internal turning process in which the boring tool advances through an already drilled hole to increase its surface finish. Boring operation is usually prone to large amount of vibrations as the boring tool is a slender component.

The boring bars have low inherent stiffness due to high length-to diameter ratio and hence are more prone to vibration. Cutting speed, feed and depth of cut are the parameters affecting the vibrational behavior of any vibrating structure. Vibration in boring operations is usually inevitable. They are unavoidable in machining, but their intensity levels can be controlled by suitable damping mechanisms, either active or passive. Even though active methods are more effective than passive methods, complexity in design and high external power consumption make it very expensive. Passive suppression mechanisms are simple in construction, low cost, and do not need external energy. Tool tip of the boring bar is the place where vibrations will occur the most and are of major concern as it is the cutting zone of the process.

The vibration attenuation improves the stiffness of boring bar._By using passive damping techniques we can control vibrations of the tool. For this purpose we use particle damping and MR fluid techniques.
An innovative vibration suppression method based on a magneto-rheological (MR) fluid-controlled boring bar for vibration suppression can change stiffness of boring bar. The stiffness of vibrating structure can be adjusted using MR-fluid controlled boring bar. Various particles like copper, silicon, lead, steel, rubber, lead carbide powder etc can be used as damping material to attenuate the vibrations which can increase the damping capability. It converts the kinetic energy of boring bar to heat wherein in other conventional operations internal strain energy is converted into heat. This is how higher amount of damping is achieved in this technique of vibration reduction.

Particle damping is remarkably effective in vibration control of boring bar. A passive damping technique is used for the boring tool design. It increases the damping ability of the boring bar in terms of damping ratio by reducing large amount of vibrations of the boring tool retaining the stiffness of the boring bar within acceptable values thereby maintaining the strength of boring bar. 


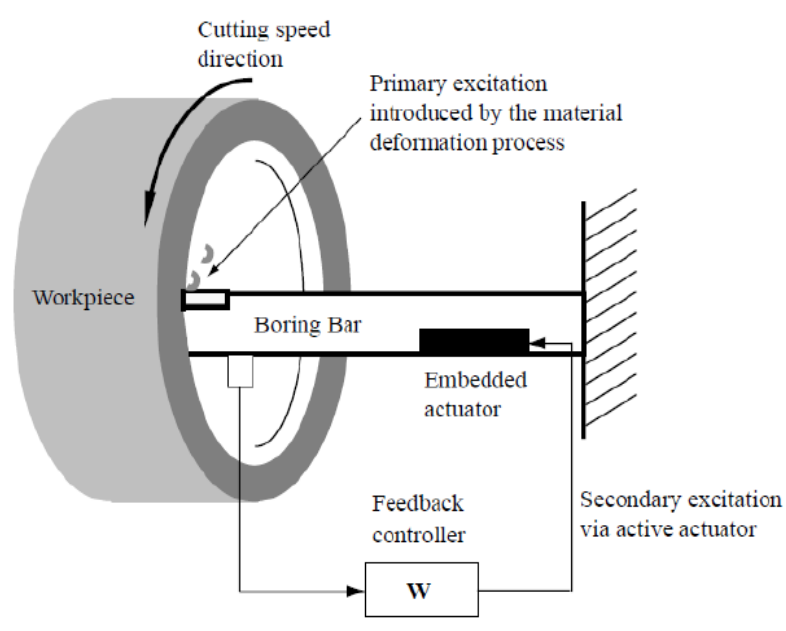

Fig 1: Schematic diagram of the boring bar [8]

\section{LITERATURE REVIEW}

M. Senthil kumar et al. [1] 2011 worked on particle damping technique for the control of vibrations in boring bar. He investigated the efficiency of particle damping in vibration attenuation of boring bar using damping particles like copper and lead. In this regard, Boring bar is drilled to have a longitudinal hole in which damping particles were embedded. Experimental investigations were carried out to find out the settling time of boring bar for different particles by giving an impact pulse by an impact hammer to the bar held as a cantilever beam. Damping performances of these particles having various sizes were observed and compared .Natural frequencies of the solid boring bar acting as a beam were compared with those of the drilled boring bar.

Displacement - time plots of boring bar without damping particles were compared with the plots with damping particles filled upto $100 \%$ volumetric packing ratio.

A set of particles yielding the best result of vibration control was determined. The dependency of the amount of damping and the frequency at which maximum damping occurs on the size of particles revealed that particles can be designed to control the vibrations in boring bar.

C. V. Biju et al. [2] focused on investigation into the effect of passive damping technique using damping particles on surface topography when boring operation is being processed. For this purpose they used a particle damping method called the novel method, for vibration control during boring process. They modeled the boring bars without cavity and with cavity and analyzed using Ansys. Spherical steel particles were used as damping particles in a cavity near machining end of the designed boring bar. Damping characteristics of the boring bar were evaluated for varying sizes and volumetric ratios of damping particles using impact and shaker tests and results were included. Results of experimentation in terms of surface roughness and chatter marks were analyzed and the results showed an improvement of bore quality with particle impact damping as compared with a boring bar without PID.
Shaker tests revealed that $3.17 \mathrm{~mm}$ sized steel damping particles with $50 \%$ volume fraction reduce the amplitude response of boring bar. They showed that the transfer of momentum from the vibratory system to the damping particles and the energy dissipation caused by collision between the particles and cavity reduce the self-excited vibration of boring bar thereby improving the stability of boring operation.

B. Moetakef-Imani et al. [3] presented the dynamic simulation of boring process. They presented a model for this simulation. They studied the causes of vibration and vibrational behavior of boring bar for certain cutting conditions and revealed that boring bar is easily subjected to vibrations because of its large slenderness ratio. They used B-spline parametric curves to simulate different tool geometries with a single approach. Euler-Bernoulli Beam theory was used for boring bar modeling. They stated that the structure comprising of lathe machine, boring tool and the work-piece undergo excessive vibrations under certain conditions.

Zhehe Yao et al. [4] worked on chatter suppression by parametric excitation. In this study, the effect of parametric excitation on a van der Pol-Duffing oscillator with a time delay feedback was studied using the averaging method. They validated the effect of parametric excitation on chatter suppression through the cutting experiments using MagnetoRheological (MR) fluid-controlled boring bar. The effect of parametric excitation on the self-excited vibration system was studied regardless of the generation mechanisms of the self-excited vibration. The regenerative effect is the most common effect that generates chatter in the machining processes. Therefore, they studied the regenerative effect for the stability analysis of the cutting vibration system. Parametric excitation effects on chatter suppression were investigated by experimental validation and theoretical analysis. The cutting experiments using magnetorheological fluid controlled boring bar showed remarkable effects on chatter suppression.

Henrik Akesson et al. [5] concentrated on studying the various clamping conditions of boring tool. They worked on the effect of different clamping properties on the dynamic properties of clamped boring bars. They discussed about Euler Bernoulli modeling of clamped boring bar with emphasis on the modeling of the clamping conditions. Experimental investigation results show variation in dynamic characteristics of boring bar as per the changes in the clamping positions of boring bar. is investigated experimentally. Standard and modified boring bars are considered. The influence of standard coupling housings with different number of clamping screws, different clamping screw diameters, different screw tightening torques, on eigen frequency values and its mode shapes orientation in cutting speed, cutting depth plane was calculated. Deqing Mei et al. [6] proposed an MR fluidcontrolled chatter suppressing boring Bar. They established a dynamic model of an MR fluid-controlled boring bar based on an Euler-Bernoulli beam model. FEM analysis 
was applied for designing the magnetic field. They analyzed the regions of operating stability using the dynamic beam model, and concluded that it can be used suppress the chatter by adjusting the damping and natural frequency of the system. Experimental results regarding the vibrations at the structure's tip in different spindle speeds validated the model and demonstrated chatter suppression in a boring process and reduced the chatter.

S. Devaraj et al. [7] proposed fine particle impact damping method in boring operation for surface quality enrichment of the workpiece. Damping to suppress the vibrations was provided by embedding fine particles within small hole of a vibrating structure. They performed experimental investigation for the surface roughness measurement of workpieces. using Copper, Aluminium, Zinc and Silicon particles but having different densities. The results they obtained proved that the usage of silicon and zinc particles shows less damping capability when compared to the damping capabilities of the boring tool using Copper and Aluminium particles. and thus it revealed that the surface finish value of the workpiece can be improved using particle impact damping.

Pranali Khatake et al. [8] introduced a vibration attenuation technique for boring bar through the implementation of passive damper. They used damping particles within the boring bar and experimental investigation was undertaken to observe the surface finish of specimen using different overhang lengths of boring bar during operation. The results proved that the chatter of the tool is suppressed at a larger amount which means the self excited vibrations of the boring tool are reduced..

Steven E. Olson et al. [9] established An analytical particle damping model. They did an analytical evaluation of the particle damper. They utilized the particle dynamics method based on the kinematics of particle damping, involving shear friction between the particles and contacting areas and the dissipation of energy in the form of heat of the particle material. Interaction forces between the individual particles and the cavity walls are calculated based on forcedisplacement relations. Application of the model has been demonstrated by simulating laboratory testing of a cantilevered beam.

Zhiwei $\mathrm{Xu}$ et al. [10] investigated a structure in which damping particles were embedded in the horizontal hole drilled in the vibrating structure. They concentrated on the study of shear of the boring bar and its effects on the damping capability of the structure. They presented an analytical model to analyze the effect of particle damping on vibration behavior of boring bar. They used different volumetric ratios of particles and also different types of particles and their various sizes to observe the damping effect of each and to suggest a good damping material for better vibration reduction. The passive damping using damping particles is proved to be effective. Although it is non-linear, it can give a strong energy dissipation rate.

\section{SUMMARY}

This review paper reveals that passive damping techniques by using Magneto-Rheological fluid (MR) and damping particles like copper, lead, silicon, rubber etc. can suppress the vibrations during boring operation to the desired amount. Particle damping is a better passive damping technique which can retain its stiffness within permissible limits even after the geometry changes of the boring tool. This proves to be a simpler and effective method of vibration reduction. This can be used over a wide range of temperatures and frequencies and shows a wide scope of applications in machining industries. This has investigated the damping enhancement method with particulate materials like damping particles and MR fluid. This suggests that the Particles and MR fluid can be designed to achieve mitigation of vibration. The review shows a greater damping capability enhancement in comparison with the conventional tool.

\section{ACKNOWLEDGEMENTS}

I am very thankful to my project guide Prof. R.V. Patil and G. S. Waghmare, Assistant professor, Mechanical Engineering Department, Sinhgad Institute of Technology and Science, Narhe, Pune, for his continuous support and encouragement in completing this work.

\section{REFERENCES}

[1]. M. S. Kumar, K. M. Mohanasundaram and B. Sathishkumar, A case study on vibration control in a boring bar using particle damping, International Journal of Engineering, Science and Technology, 2011, 3 (8), pp. 177184.

[2]. Kanase Sandip S1,Patil Jaydeep S2 ,Jadhav Sainand M3, Improvement Of Ra Value Of Boring Operation Using Passive Damper, The International Journal Of Engineering And Science (IJES), 2013, 2(7),pp. 103-108.

[3]. B. Moetakef-Imani , N.Z. Yussefian, Dynamic simulation of boring process, International Journal of Machine Tools \& Manufacture, 2009, 49,pp.1096-1103.

[4]. Zhehe Yao, Deqing Mei, Zichen Chen, Chatter suppression by parametric excitation: Model and experiments, Journal of Sound and Vibration, 2011,330,pp 2995-3005.

[5]. Henrik Akesson, Tatiana Smirnova, Lars Hakansson, Analysis of dynamic properties of boring bars concerning different clamping conditions, Mechanical Systems and Signal Processing, 2009, 23, pp. 2629-2647.

[6]. Deqing Mei, Tianrong Kong, Albert J. Shih, Zichen Chen, Magneto-rheological fluid-controlled boring bar for chatter suppression, journal of materials processing technology,2009, 209, 1861-1870.

[7]. S. Devaraj1, D. Shivalingappa, Channankaiah, Rajesh S Jangaler4, Surface Quality Enrichment Using Fine Particle Impact Damper In Boring Operations, International Journal of Research in Engineering and Technology, 2014, 03(02), pp.531-535. 
[8]. Pranali Khatake1, P. T. Nitnaware, Vibration mitigation using passive damper in machining, International Journal of Modern Engineering Research (IJMER), 2013, 3( 6), pp3649-3652.

[9]. Steven E. Olson, An analytical particle damping model, Journal of Sound and Vibration 264 (2003) 1155-1166.

[10]. Zhiwei Xua, Michael Yu Wangb, Tianning Chenc, Particle damping for passive vibration suppression: numerical modeling and experimental investigation, Journal of Sound and Vibration, 2009, 207, pp. 1097-1120. 